\title{
Distribuição regional do suor durante exercício progressivo até a fadiga
}

CDD. 20.ed. 612.7921

796.022

\author{
João Batista FERREIRA JÚNIOR* \\ Kenya Paula Moreira OLIVEIRA* \\ Michele Atalla da FONSECA* \\ Leonardo Gomes Martins COELHO* \\ Flávio de Castro MAGALHÃES ${ }^{* * * *}$ \\ Valéria França COSTA** \\ Angelo Ruediger Pisani MARTINI* \\ Renata Lane de Freitas PASSOS* \\ Francisco Teixeira COELHO* \\ Luiz Oswaldo Carneiro RODRIGUES*
}

*Escola de Educação
Física, Fisioterapia e
Terapia Ocupacional,
Universidade Federal
de Minas Gerais.
**Faculdade de Farmá-
cia, Universidade Fe-
deral de Minas Gerais.
***Escola de Educa-
ção Física e Esporte,
Universidade de São
Paulo.

\section{Resumo}

A partir da hipótese evolutiva de que a cabeça humana precisa ser resfriada de forma especial, o objetivo do presente estudo foi verificar se a sudorese seria maior na testa do que nas demais regiões do corpo durante o exercício progressivo até a fadiga (EPF). Em 17 voluntários ( $23 \pm 2$ anos, 76,93 $\pm 7,74$ $\mathrm{kg}, 179 \pm 7 \mathrm{~cm}$ e 1,9 $\left.\pm 0,1 \mathrm{~m}^{2}\right)$ foram medidos a taxa de sudorese local ( IS $_{\text {local }}$ ), o número de glândulas de suor ativas (GSA) e taxa de suor por GSA (TS local $^{\circ} \mathrm{GSA}^{-1}$ ) em oito regiões do corpo (testa, costas, peito, braço, antebraço, mão, coxa e perna) durante o EPF em cicloergômetro. A TSlocal da testa foi maior que todas as outras regiões e a $\mathrm{TS}_{\text {local }}$ do peito foi maior apenas que a da coxa. 0 número de GSA da testa foi maior do que em todas as outras regiões, e a GSA da mão foi maior que do peito, braço, coxa e perna. $\mathrm{A} \mathrm{TS}_{\text {local }} \mathrm{GSA}^{-1}$ da testa foi maior do que as do braço, antebraço, mão e coxa, e a $\mathrm{TS}_{\text {local }} \mathrm{GSA}^{-1}$ das costas e do peito foram maiores que do antebraço e mão. A produção de suor da parte superior do corpo (testa, costas, peito, braço, antebraço, mão) foi maior que a inferior (coxa e perna). Concluiu-se que o EPF desencadeou um padrão de produção de suor maior na cabeça que pode estar relacionado à maior convecção nas regiões mais altas do corpo.

UnItermos: Sudorese; Exercício; Fadiga.

\section{Introdução}

A capacidade humana de suar é um dos principais mecanismos utilizados para manutenção da temperatura corporal durante o exercício, principalmente no ambiente quente e seco. Sabe-se que diversos fatores influenciam a capacidade de produzir suor como o estado de aclimatação ao calor (Machado-Moreira, MagalHães, Vimieiro-Gomes, Lima \& Rodrigues, 2005; Nadel, Pandolf, Roberts \& Stolwijk, 1974; Patterson, Stocks \& Taylor, 2004), a capacidade aeróbica (BuONo \& SJOHOLM, 1988; NADEL et al., 1974), o estado de hidratação (ForTNEY, Nadel, Wenger \& Bove, 1981; Sawka, Gonzalez, Young, Dennis, Valeri \& Pandolf, 1989), diferenças sexuais (Madeira, 2007; Morimoto, Slabochova, Naman \& Sargent, 1967; Rosa, 2005; Sá, 2004; Weinman, Slabochova, Bernauer, Morimoto \& Sargent, 1967) e a idade (Fonseca, 2007; Inoue, Havenith, Kenney, LOOMIS \& Buskirk, 1999; TANKERSLEy, SMOLANDer, Kenney \& Fortney, 1991). Os fatores que influenciam a capacidade humana de suar têm 
sido temas de estudo da termorregulação, sendo um deles a diferença na produção de suor entre as regiōes do corpo. A TABELA 1 mostra os estudos realizados com essa temática.

TABELA 1 - Estudos realizados da diferença na taxa de suor (TS) entre as regiões do corpo.

\begin{tabular}{|c|c|c|}
\hline Autores & Protocolo & Resultados \\
\hline $\begin{array}{l}\text { PatTerson, Stocks } \\
\text { e TAYLOR }(2004)\end{array}$ & Aclimatação ao calor. & $\mathrm{TS}_{\text {peito }}>\mathrm{TS}_{\text {coxa e antebraço }}>\mathrm{TS}_{\text {coxa e testa. }}$. \\
\hline Kondo et al. (1998) & $\begin{array}{l}\text { Exercício a } 35,50 \text { e } 65 \% \text {. } \\
\mathrm{VO}_{2 \max }, 30{ }^{\circ} \mathrm{C} \text { e } 42 \% \text { URA. }\end{array}$ & $\begin{array}{l}\text { A } 35 \% \text { do } \mathrm{VO}_{2 \max } \text { a } \mathrm{TS}_{\text {testa e peito }}>\mathrm{TS}_{\text {costas, antebraço e coxas }} \\
{\text { A } 65 \% \mathrm{VO}_{2 \max } \text { a TS }}_{\text {testa }}>\mathrm{TS}_{\text {peito }}\end{array}$ \\
\hline $\begin{array}{l}\text { Cotter, PatTerson } \\
\text { e TAYlor (1997) }\end{array}$ & $\begin{array}{l}\text { Exercício a } 50 \% \mathrm{VO}_{2 \max } \\
40{ }^{\circ} \mathrm{C} \text { e } 60 \% \text { URA. }\end{array}$ & $\mathrm{TS}_{\text {testa e máo }}>\mathrm{TS}_{\text {costas, braço, antebraçọ, perna coxa e pé́ }}$ \\
\hline $\begin{array}{l}\text { CotTer, PatTERson } \\
\text { e TAYLOR (1995) }\end{array}$ & $\begin{array}{l}\text { Exercício a } 40 \% \text { da potência } \\
\text { máxima a } 37^{\circ} \mathrm{C} \text { e } 46 \% \text { URA. }\end{array}$ & $\begin{array}{l}\mathrm{TS}_{\text {testa }}>\mathrm{TS}_{\text {peito, perna e panturrilha }} \\
\mathrm{TS}_{\text {costas }}>\mathrm{TS}_{\text {coxa e panturrilha }}\end{array}$ \\
\hline $\begin{array}{l}\text { InOuE et al. (1991, } \\
1995)\end{array}$ & $\begin{array}{l}\text { Pernas submersas em água } \\
\text { quente }\left(43^{\circ} \mathrm{C}\right) .\end{array}$ & $\mathrm{TS}_{\text {máo }}>\mathrm{TS}_{\text {coxa }}$, em jovens e idosos. \\
\hline $\begin{array}{l}\text { NADEL, BulLARD e } \\
\text { STOLWIJK (1971) }\end{array}$ & $\begin{array}{l}\text { Exercício a } 80 \% \mathrm{VO}_{2 \max } \\
26^{\circ} \mathrm{C} \text { e } 80 \% \text { URA. } \\
\text { Não foi reallizada análise } \\
\text { estatística. }\end{array}$ & $\mathrm{TS}_{\text {costas }}>\mathrm{TS}_{\text {peito }}>\mathrm{TS}_{\text {abdômen }}>\mathrm{TS}_{\text {coxa }}>\mathrm{TS}_{\text {antebraço }}$ \\
\hline Sato e Dobson (1970) & $\begin{array}{l}\text { Repouso a } 47^{\circ} \mathrm{C} \text { e } 80-90 \% \\
\text { URA. }\end{array}$ & $\mathrm{TS}_{\text {testa }}>\mathrm{TS}_{\text {máo e antebraço }}$ \\
\hline HERTZMAN (1957) & Repouso a 35 e $48^{\circ} \mathrm{C}$. & $\mathrm{TS}_{\text {panturrilha }}>\mathrm{TS}_{\text {coxa }}>\mathrm{TS}_{\text {abdômen }}>\mathrm{TS}_{\text {peito }}$ \\
\hline Kuno (1956) & & $\begin{array}{l}\mathrm{TS}_{\text {peito e costas }}>\text { TS dos membros inferiores. } \\
\mathrm{TS}_{\text {testa }}>\mathrm{TS} \text { dos membros superiores. } \\
\text { A TS dos membros superiores e inferiores aumenta- } \\
\text { ram das partes proximais para distais. }\end{array}$ \\
\hline HERTZMAN et al. (1952) & Repouso a 26,30 e $36{ }^{\circ} \mathrm{C}$. & $\begin{array}{l}\text { A } 26^{\circ} \mathrm{C} \text { a TS } S_{\text {testa }}>\mathrm{TS}_{\text {peito, abdômen, panturilha e coxa. }} \\
\text { A } 36^{\circ} \mathrm{C} \text { não houve diferença entre as regióes. }\end{array}$ \\
\hline WEINER (1945) & $\begin{array}{l}\text { Exercício em ambiente } \\
\text { quente até atingir } \\
\text { temperatura interna de } 39^{\circ} \mathrm{C} \text {. }\end{array}$ & $\begin{array}{l}\text { A TS } \\
\text { regiôesta e tronco }\end{array}$ \\
\hline
\end{tabular}

Hertzman, Randall, Peiss e Seckendorf (1952), Kondo, Takano, Aoki, Shibasaki, Tominaga e InOUe (1998), PATTERSON, StOcks e TAYlor (2004), SATO e Dobson (1970), encontraram taxa de suor da testa maior que nas outras regióes do corpo. Cotter, Patterson e Taylor (1995), Inoue, NaKaO, AraKi e Murakami (1991), Kuno (1956), Nadel, Bullard \& Stolwijk (1971), Patterson, STOCKS e TAYLOR (2004), WEINER (1945) verificaram maior produção de suor na região superior do corpo que na inferior. Entretanto, os resultados existentes mostram um padrão heterogêneo da distribuição regional do suor, já que são variados e algumas vezes contraditórios, o que pode ser devido aos diferentes protocolos usados (estresse térmico, exercício e repouso, locais de medida do suor) e às diferenças individuais observadas por muitos autores (COTTER,
Patterson e Taylor, 1995; Hertzman, 1957; Kuno, 1956; Nadel, Bullard \& STOlWIJK, 1971; SATO \& Dobson, 1970). De acordo com os conceitos clássicos de KunO (1956), a distribuição regional da sudorese em áreas mais expostas à convecção favoreceria a dissipação de calor, fazendo com que o suor seja profuso nas regiōes onde a evaporação for facilitada. Entretanto, poucos estudos têm abordado esta questão de maneira quantitativa.

A maioria dos estudos sobre a sudorese humana durante o exercício tem utilizado protocolos de exercícios contínuos (COTTER, Patterson e Taylor, 1995, 1997; Kondo et al., 1998; NAdel, Bullard \& STOLWIJK, 1971; Patterson, StOcks e Taylor, 2004; WeINER, 1945), nos quais os estímulos para a produção de suor podem ser predominantemente térmicos, já que estão mais relacionados à produção 
de calor metabólico e ao estresse térmico do ambiente (Amorim, Rodrigues, Ribeiro, Prado, Bonfim \& OliveIra, 1999), do que não térmicos, ou seja, relacionados à estimulação simpática, a qual é proporcional à intensidade relativa do exercício (AMORIM, 2003; AMORIM, ViMIEIRO-GOMES, Machado-Moreira, Magalhāes, Rosa, Prado \& RODRIGUES, 2006). O exercício progressivo até a fadiga (EPF) tem se mostrado um método sensível na medição da produção de suor (MACHADO-MOREIRA et al., 2005; Vimieiro-Gomes, Magalhäes, AMorim, Machado-Moreira, Rosa, Lima \& Rodrigues, 2005). Esse tipo de exercício, com duração média de aproximadamente 20 minutos, provoca produção de suor através de estímulos térmicos e não térmicos (ShibASAKI, Kondo \& CRANDAll, 2003). Entretanto, não se sabe se os estímulos térmicos e não térmicos do EPF provocariam a distribuição de suor como aquela observada nos exercícios contínuos. Portanto, o objetivo do presente estudo foi verificar a hipótese de KunO (1956), de acordo com a qual o EPF deveria desencadear um padrão de distribuição de suor que seria maior na testa, por ser anatomicamente uma das áreas mais ventiladas.

\section{Procedimentos e métodos}

\section{Voluntários}

O estudo foi aprovado pelo Comitê de Ética em Pesquisa da Santa Casa de Misericórdia de Minas Gerais (012/2005), e os voluntários leram e assinaram o termo de consentimento livre e esclarecido.

Os experimentos foram realizados no Laboratório de Fisiologia do Exercício, localizado numa região tropical (latitude $19,5^{\circ} \mathrm{S}$ e longitude $43^{\circ} \mathrm{O}$ ) no hemisfério sul entre os meses de outubro e novembro de 2005 , período compreendendo a primavera, sempre de 14:00 às 17:00 horas.

Dezessete homens jovens sadios e fisicamente ativos realizaram o EPF. As características físicas dos voluntários (idade, peso, estatura, percentual de gordura do corpo (\% de gordura) calculado a partir da espessura das dobras cutâneas do bíceps, subescapular, tríceps, peitoral, subaxilar, suprailíaca, região média da coxa e região média da perna (adipômetro, Lange, Santa Cruz, CA, USA) e área de superfície corporal - ASC (Dubois \& Dubois, 1916) estão descritas na TABELA 2.

TABELA 2 - Característica físicas dos voluntários.

\begin{tabular}{ccccc}
\hline Idade (anos) & Peso $(\mathrm{kg})$ & Estatura $(\mathrm{cm})$ & ASC $\left(\mathrm{m}^{2}\right)$ & $\%$ de gordura \\
\hline $23 \pm 2$ & $76,93 \pm 7,74$ & $179 \pm 7$ & $1,9 \pm 0,1$ & $16 \pm 5$ \\
\hline
\end{tabular}

Os voluntários foram instruídos a evitarem o consumo de tabaco, cafeína, bebida alcoólica ou medicamentos e se absterem de atividade física $24 \mathrm{~h}$ antes do experimento e foram orientados a beber $500 \mathrm{ml}$ de água $2 \mathrm{~h}$ antes do experimento para garantir o estado de hidratação, que foi avaliado assim que o voluntário chegava ao laboratório por meio da medida da gravidade específica da urina (refratômetro, JSCP- Uridens®, São Paulo, Brasil), a qual foi sempre menor que 1,030 em todos os experimentos. Durante o experimento, os voluntários vestiam shorts, meias e tênis esportivo.

\section{Procedimentos}

O estudo foi conduzido em única situação, na qual foi realizado o EPF em cicloergômetro de acordo com o Colégio Americano de Medicina do Esporte (ACSM, 2000): ciclo ergômetro (Monark, 824E, Varberg, Sweden) com carga inicial de $50 \mathrm{~W}$ e aumento de $25 \mathrm{~W}$ a cada $2 \mathrm{~min}$. $\mathrm{O}$ exercício foi interrompido quando o sujeito, fisicamente ou verbalmente, desejava parar devido à fadiga. Como parte do protocolo, após a fadiga, os voluntários pedalavam por $2 \mathrm{~min}$ com $50 \mathrm{~W}$ e depois repousavam por $3 \mathrm{~min}$. Os voluntários foram pesados nus antes do exercício e imediatamente após a recuperação (Filizola $®$ MF-100 escala, precisão de 0,02 kg, São Paulo, SP, Brasil). A taxa de sudorese total $\left(\mathrm{TS}_{\text {total }}\right)$ foi medida pela mudança de peso corporal antes e após o exercício, relativizada pela área de superfície corporal do voluntário e dividida pelo tempo entre as pesagens, não corrigida pelas variaçôes metabólicas ou respiratórias. A taxa de sudorese local $\left(\mathrm{TS}_{\text {local }}\right)$ foi medida usando papel filtro absorvente de $9 \mathrm{~cm}^{2}$ (J Prolab, S. J. dos Pinhais, PR, Brasil) colocado em oito regiōes: região média da testa (no meio da testa), peito (no peito direito, na linha do mamilo e clavícula), na parte superior das costas (na borda superior da escápula direita), braço (parte de cima da lateral do braço direito) antebraço (parte proximal do antebraço direito), dorso da mão (no meio do dorso da mão direita), parte 
anterior da coxa (no meio da coxa) e perna (lateral da panturrilha direita). Para prevenir a evaporação do suor, o papel filtro absorvente foi coberto com pedaço de plástico de $64 \mathrm{~cm}^{2}$ fixado na pele com fita adesiva impermeável (ViMIEIRO-Gomes et al., 2005). A TSlocal foi calculada pela mudança no peso do papel filtro absorvente antes e após a colheita do suor (Mettler Toledo AB 204 escala analítica, precisão de 0,1 mg, Columbus, OH, USA) dividido pela área do papel filtro absorvente e pelo tempo de colheita do suor $\left(\mathrm{mg} \cdot \mathrm{cm}^{-2} \cdot \mathrm{min}^{-1}\right)$. A taxa de suor da parte superior do corpo foi calculada pela média da $\mathrm{TS}_{\text {local }}$ da testa, costas, peito, braço, antebraço e mão; já a taxa de suor da parte inferior do corpo foi calculada pela média da $\mathrm{TS}_{\text {local }}$ da coxa e perna. O número de glândulas de suor ativas (GSA) foi medido por meio da técnica do papel de $16 \mathrm{~cm}^{2}$ impregnado com iodo (Buono \& Sjoholmo, 1998), que era colocado no local de medida da sudorese imediatamente após o final do exercício e posteriormente contado por três pesquisadores. O número de GSA ativas foi dado em GSA. $\mathrm{cm}^{-2}$. A taxa de suor local por glândula de suor ativa $\left(\mathrm{TS}_{\text {locel }} \mathrm{GSA}^{-1}\right)$ foi calculada pela divisão da taxa de suor local pelo número de glândulas de suor ativas (mg.glând $\left.{ }^{-1} \cdot \mathrm{min}^{-1}\right)$. Em adição, a frequência cardíaca

\section{Resultados}

Ao final do exercício, com tempo total de 15,36 \pm $3,43 \mathrm{~min}$, a PSE teve média de $19 \pm 1, \mathrm{FC}$ de $188 \pm 11$ bpm, mostrando que o exercício foi realizado até a fadiga. Em adição, os voluntários alcançaram potência máxima de $225 \pm 45 \mathrm{~W}$ correspondente ao $\mathrm{VO}_{2 \max }$ de $39,5 \pm 6,2$ ml. $\mathrm{kg}^{-1} \cdot \mathrm{min}^{-1} \mathrm{eTS}$ total de $3,8 \pm 1,1 \mathrm{~g} \cdot \mathrm{cm}^{-2} \cdot \mathrm{min}^{-1}$.

A FIGURA 1 mostra a taxa de sudorese medida nas oito regiōes durante o EPF. A TS local $_{\text {da }}$ testa foi maior que todas as outras regióes, já a $\mathrm{TS}_{\text {local }}$ do peito foi maior apenas que da coxa.

A FIGURA 2 mostra o número de GSA medido logo após o término do EPF. O número de GSA da
(FC) (Polar Vantage NV, Kempele, Finlândia) e escala de percepção subjetiva de esforço (PSE) de BORG (1982) foram medidas a cada 2 min.

Os procedimentos foram realizados numa sala com as seguintes condições térmicas ambientais: $22 \pm 0{ }^{\circ} \mathrm{C}$ e $64 \pm 12 \%$ de umidade relativa, mantido através de ar condicionado comercial (Cônsul AirMaster 7500, São Paulo, SP, Brasil).

\section{Análise estatística}

Foi realizada análise descritiva da amostra, sendo calculados: média, desvio padrão, normalidade e homogeneidade. Como as variáveis não apresentaram normalidade e ou homogeneidade, elas foram transformadas: a produção de suor, GSA, TS.GSA-1 pela fórmula: $\log (\mathrm{x}+1)$, e a taxa de suor da parte superior e inferior do corpo pela fórmula: $\log (\mathrm{x})$. A comparação das variáveis: produção de suor, GSA, produção de suor. GSA-1 entre as regiōes do corpo foram realizadas pela análise de variância "one-way", e as diferenças foram detectadas pelo "post- hoc" de Tukey. A comparação da taxa de suor entre a região superior e inferior do corpo foi comparada pelo teste $t$ de student. O nível de significância adotado foi de 5\%.

testa foi maior que todas as outras regiões, exceto mão, que foi maior que do peito, braço, coxa e perna.

A FIGURA 3 mostra a $\mathrm{TS}_{\text {local }}$ GSA ${ }^{-1}$ calculada pela razão entre a $\mathrm{TS}_{\text {local }}$ e GSA ${ }^{-1}$ durante o EPF. A $\mathrm{TS}_{\text {local }} \mathrm{GSA}^{-1}$ da testa foi maior que do braço, antebraço, mão e coxa. A TS local $\mathrm{GSA}^{-1}$ das costas e do peito foram maiores que do antebraço e mão.

A FIGURA 4 mostra a taxa de sudorese do membro superior e do membro inferior durante o EPF. A produção de suor da parte superior do corpo foi maior que a inferior. 


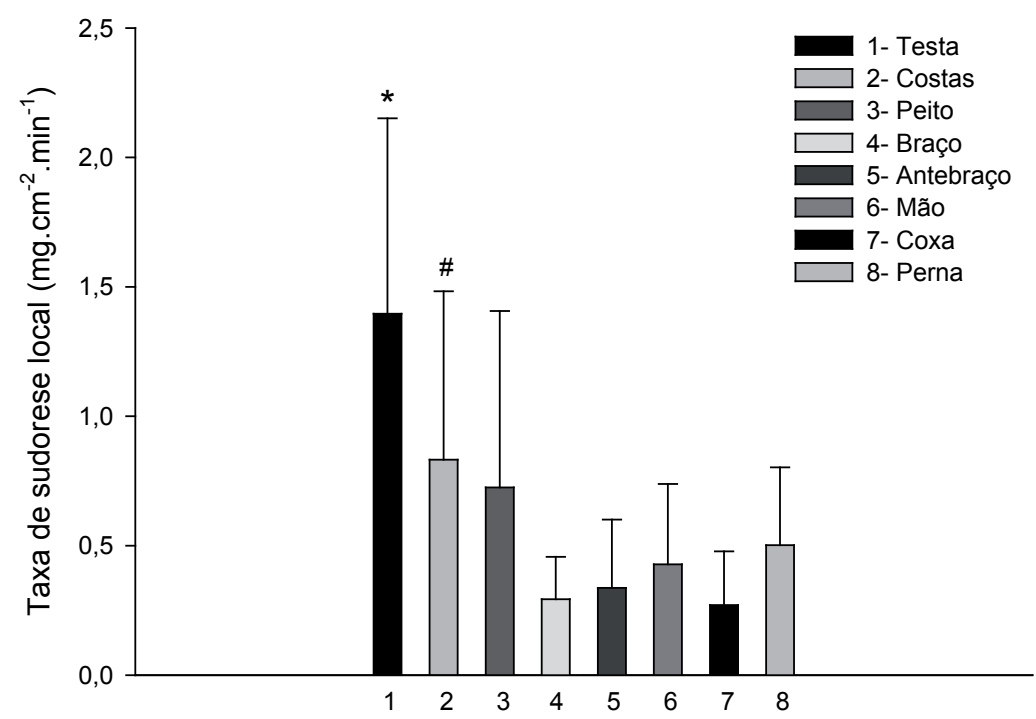

${ }^{*} p<0,05$, diferente das outras regiões.

$\# p<0,05$, diferente de coxa.

FIGURA 1 - Taxa de sudorese local durante exercício progressivo até a fadiga.

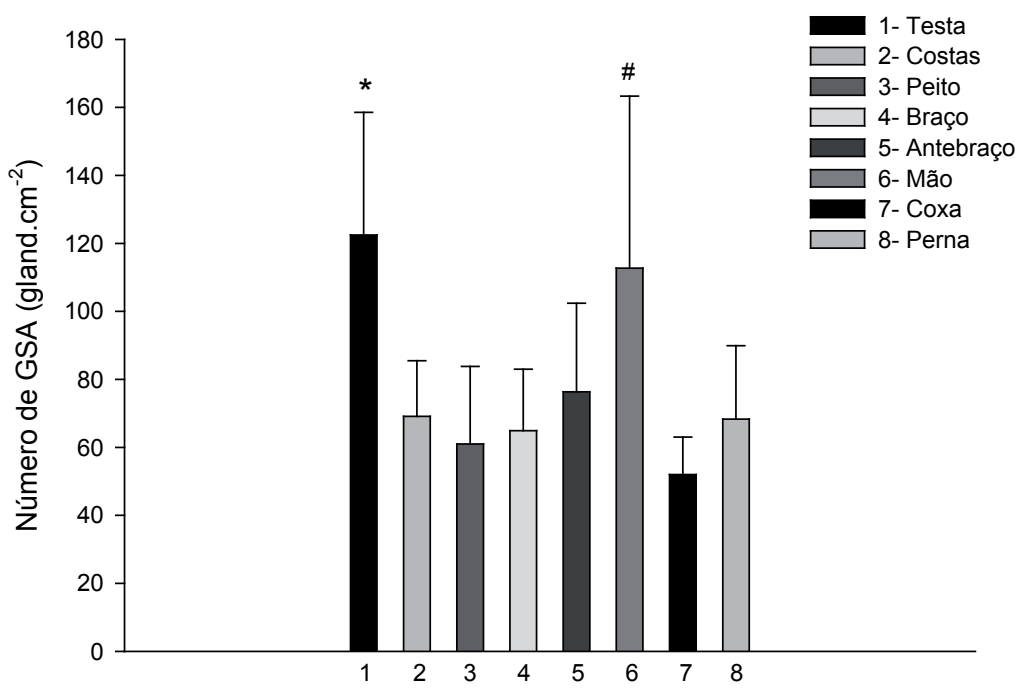

${ }^{*} p<0,05$, diferente das outras regiões.

$\# p<0,05$, diferente de peito, braço, coxa e perna.

FIGURA 2 - Número de glândulas de suor ativas durante exercício progressivo até a fadiga. 
${ }^{*} p<0,05$, diferente de braço, antebraço, mão e coxa.

$\# p<0,05$, diferente de antebraço e mão.

${ }^{*} p<0,05$, diferente da parte inferior do corpo.

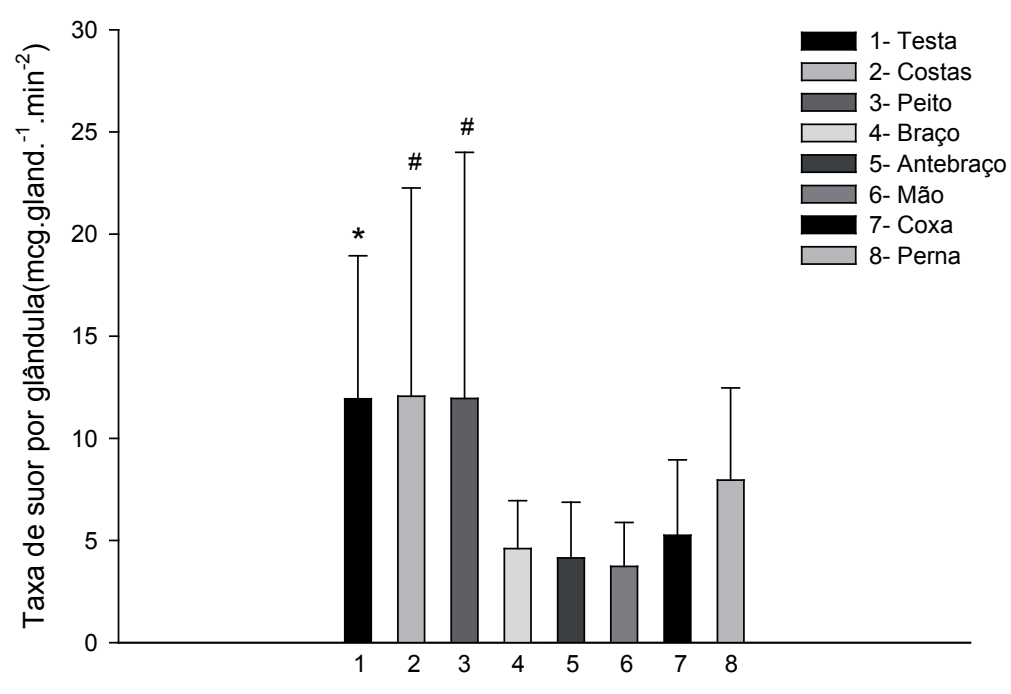

FIGURA 3 - Taxa de suor por glândula durante exercício progressivo até a fadiga.

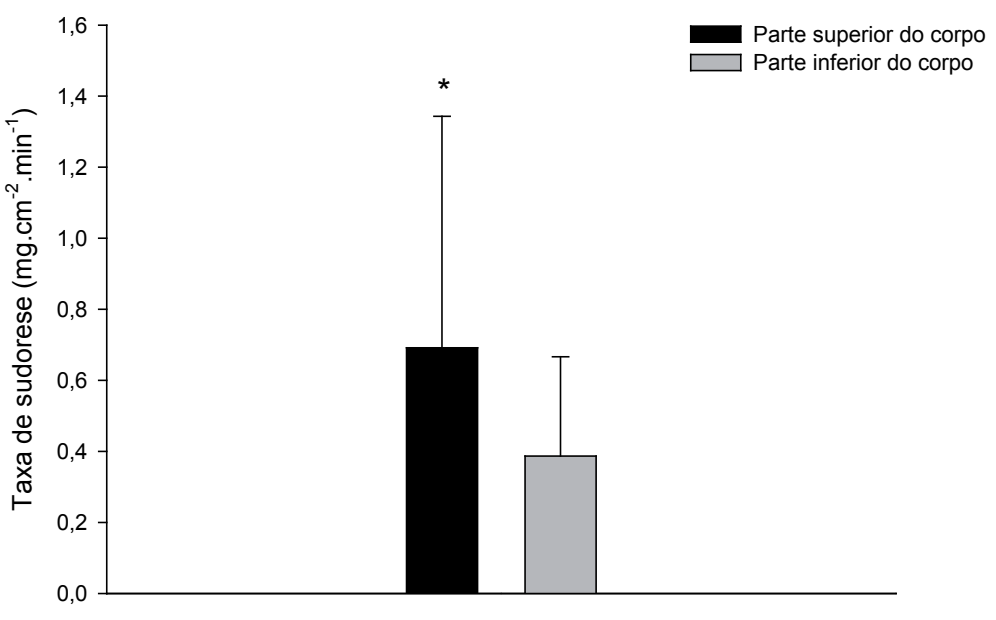

FIGURA 4 - Taxa de sudorese da parte superior e inferior do corpo durante exercício progressivo até a fadiga.

\section{Discussão}

O presente estudo encontrou maior produção de suor na testa comparado às outras regiōes do corpo, o que sugere que a distribuição regional da sudorese em áreas mais expostas à convecção favoreceria a dissipação de calor (KunO, 1956), portanto, um modelo aparentemente aplicável ao EPF. Apesar dos diferentes protocolos usados, esse achado corrobora os de outros estudos que utilizaram exercício contínuo (Hertzman et al., 1952; Kondo et al., 1998; Patterson, Stocks \& Taylor, 2004; Sato \& Dobson,
1970). Além disso, verificou-se maior GSA na testa do que nas outras regiōes do corpo, exceto na mão; maior $\mathrm{TS}_{\text {local }}$ GSA-1 na testa comparado com braço, antebraço, mão e coxa; e maior TS na parte superior do corpo comparado à parte inferior. Estes resultados indicam que o EPF parece desencadear um padrão de distribuição de suor entre as regiōes do corpo, no qual a cabeça sua mais que as demais regióes.

Sabe-se que a sudorese é ativada por estímulos eferentes oriundos do hipotálamo, mais especificamente 
da área pré-óptica, em função de estímulos térmicos e não térmicos (Shibasaki, Kondo \& Crandall, 2003). O estímulo hipotalâmico aumenta a produção de suor de forma proporcional à temperatura corporal interna (como resultado do metabolismo interno), possivelmente à temperatura cerebral e à temperatura da pele (como resultado do estresse térmico ambiental) (NADEL, Bullard \& STOLWIJK, 1971; Nadel, Mitchell, Saltin \& StolWijK, 1971). Os estímulos não-térmicos, tais como o estado emocional, atividades mentais e fatores relacionados ao exercício (ativação do comando central, mecano e metaboreceptores) também iniciam as respostas sudoríparas, e estas respostas são dissociadas da variação na temperatura corporal (KunO, 1956; Shibasaki, Kondo \& Crandall, 2003). É possível que a produção de suor observada no presente estudo durante a realização do EPF tenha sido o resultado da estimulação neural originada dos mecanismos térmicos e não-térmicos, já que o presente exercício teve tempo máximo de vinte e dois minutos, tempo de exercício considerado insuficiente para a elevação expressiva da temperatura interna (EIKEN \& Mekjavic, 2004; Vimieiro-Gomes et al., 2005).

É provável que os estímulos não-térmicos que podem ter contribuído para o início das respostas sudoríparas no presente estudo tenham sido a ativação do comando central, e os reflexos provenientes dos mecano e metaboreceptores. A participação de metaboreceptores na resposta sudorípara foi demonstrada por SHIBASAKI et al. (2003). Utilizan do exercício isométrico seguido de oclusão arterial pós-exercício, com e sem bloqueio neuromuscular parcial, esses autores constataram que a taxa de sudorese aumentou no peito e no braço contralateral, durante o exercício, mesmo sem quaisquer alteraçôes na temperatura interna e da pele. Ainda, a taxa de sudorese permaneceu aumentada no período de isquemia pósexercício voltando imediatamente ao valor basal após a liberação do fluxo sanguíneo. Por último, na situação com bloqueio neuromuscular parcial, a sudorese aumentou durante o exercício mesmo quando a força exercida foi próxima de zero, mas voltou aos valores basais logo após, durante a isquemia. Além disso, estudos com movimentação passiva e ativa dos membros, sugerem que metaboreceptores também são capazes de modular a sudorese, mesmo que de forma bem menos determinante que o comando central e que os metaboreceptores (SHIBASAKI, WILSON \& Crandall, 2006). Esses resultados mostram que tanto o comando central quanto neurônios metabolicamente e mecanicamente sensíveis contribuem para a resposta sudorípara durante o exercício.
Algumas consideraçōes podem ser feitas acerca da distribuição regional da sudorese a partir dos resultados do presente estudo e de outros já realizados: a) invariavelmente, os estudos que mediram a sudorese da testa verificaram que esse foi o local que mais suou comparado com as outras regióes do corpo (Hertzman et al., 1952; Kondo et al., 1998; Patterson, Stocks \& TAYlor, 2004; Sato \& DobSON, 1970) ou apresentou sudorese similar aos locais com maior taxa de suor (COTTER, Patterson \& TaYlor, 1995, 1997; Kuno, 1956; WEINER, 1945); b) estudos que compararam a taxa de suor da região superior com a inferior do corpo observaram que a taxa de suor da região superior foi maior (Cotter, Patterson \& TAYlor, 1995; Inoue et al., 1991; Kuno, 1956; Nadel, Bullard \& Stolwijk, 1971; Patterson, Stocks \& Taylor, 2004; WeINER, 1945); c) regióes que teriam maior convecção durante a corrida também parecem apresentar maior taxa de suor, como a testa (HERTZMAN et al., 1952; Kondo et al., 1998; PATTERSOn, StOCKS \& TaYlor, 2004; Sato \& Dobson, 1970), peito (Kondo et al., 1998; Kuno, 1956; WeIner, 1945), antebraço (Kuno, 1956) e dorso da mão (CotTer, Patterson \& Taylor, 1997; Kuno, 1956). Portanto, é necessário discutir um modelo que justifique a distribuição regional da sudorese.

De acordo com PORTER (1993), a velocidade do vento aumenta e a temperatura do ambiente reduz de acordo com escala logarítmica da altura em relação ao solo. Sendo assim, seria mais interessante para um animal bípede suar mais nas regióes mais altas do corpo, dado que a evaporação do suor depende da velocidade do vento. Seguindo esse mesmo raciocínio, também seria interessante para um animal ativo durante o período quente do dia, suar nas regiōes de maior convecção durante o exercício físico. Então, do ponto de vista teórico, o homem deveria ser capaz de suar nas regiões de superfícies mais expostas, nas regiōes mais altas e nas áreas de maior convecção durante o estresse térmico. $\mathrm{O}$ padrão de distribuição do suor encontrado nesse estudo parece dar suporte a essa hipótese, já que o suor da parte superior do corpo representou $64 \%$ do suor total produzido. Esse percentual está próximo dos $75 \%$ encontrado por Kuno (1956) e WeINER (1945), e essa região representa $60 \%$ da área de superfície corporal (Weiner, 1945). Além disso, alguns estudos mostram que a taxa de suor do antebraço (KunO, 1956), peito (Kondo et al., 1998; KunO, 1956; Weiner, 1945), dorso da mão (CotTer, PATterson \& TaYlor, 1997; Kuno, 1956) e testa (CotTer, 
Patterson \& TaYlor, 1995, 1997; Hertzman et al., 1952; Kondo et al., 1998; KunO, 1956; PATTERSON, STOCKS \& TaYlor, 2004; SaTo \& DobSon, 1970; WeIner, 1945), áreas de maior convecção durante a corrida, foram maiores que os outros locais. Por outro lado, o resfriamento predominante da cabeça sugere a importância desta região para o sistema termorregulatório, abrindo novas possibilidades de estudo na área da termorregulação.

Em conclusão, o EPF desencadeou um padrão de produção de suor que pode ser devido a maior convecção nas regiōes mais altas do corpo.

\begin{abstract}
Sweat regional distribution during progressive exercise until fatigue

Considering the hypothesis of human selective brain cooling during exercise should depend on greater sweating mechanism in the forehead. The purpose of this study was verify variations of sweat production between body regions during progressive exercise until fatigue (PEF). Seventeen subjects ( $23 \pm 2$ years old, $76.93 \pm 7.74 \mathrm{~kg}, 179 \pm 7 \mathrm{~cm}$ and $\left.1.9 \pm 0.1 \mathrm{~m}^{2}\right)$ volunteered for this study. Local sweat rate $\left(\mathrm{ST}_{\text {loca }}\right)$, number of active sweat glands (ASG) and sweat rate for $A S G\left(S_{\text {local }} \cdot\right.$ ASG $\left.^{-1}\right)$ in eight body regions (forehead, back, chest, arm, forearm, hand, leg and calf) were measured during PEF in cyclergometer. The $\mathrm{ST}_{\text {local }}$ of the forehead was higher than in all others regions and the chest STlocal was higher only than the leg. The number of ASG in the forehead was greater than in all other regions, and the ASG of the hand was higher than those of the chest, arm, leg and calf. The $\mathrm{ST}_{\text {local }} \mathrm{ASG}^{-1}$ of the forehead was higher than the arm, forearm, hand and leg, and the $\mathrm{ST}_{\text {local }} \mathrm{ASG}^{-1}$ of the chest and back were higher than the forearm and hand. The sweat production of the upper body (forehead, back, chest, arm, forearm and hand) was bigger than the lower body (leg and calf). In conclusion, the PEF promoted a sweating pattern in body regions that can be related to the higher convection present in the upper regions of the body.

UnITERMS: Sweat; Exercise; Fadigue.
\end{abstract}

\title{
Referências
}

AMERICAN COLLEGE OF SPORTS MEDICINE. Position stand: the prevention of thermal injuries during distance running. Medicine and Science in Sports and Exercise, Hagerstown, v.19, p.529-33, 2000.

AMORIM, F.T. Estudo da taxa de sudorese durante exercício intermitente comparada com a indução da sudorese com pilocarpina. 2003. 98 f. Dissertação (Mestrado em Educação Física) - Escola de Educação Física, Fisioterapia e Terapia Ocupacional, Universidade Federal de Minas Gerais, Belo Horizonte, 2003.

AMORIM, F.T.; RODRIGUES, L.O.C.; RIBEIRO, G.A.; PRADO, L.S.; BONFIM, I.P.; OLIVEIRA, A. Cálculo da reidratação em função da intensidade do exercício e das condições térmicas ambientais. In: FÓRUM NACIONAL DO ESPORTE, 1., 1999, Belo Horizonte. Anais... Belo Horizonte: [s.n.], 1999.

AMORIM, F.T.; VIMIEIRO-GOMES, A.C.; MACHADO-MOREIRA, C.A.; MAGALHÃES, F.C.; ROSA, M.S.; PRADO, L.S.; RODRIGUES, L.O.C. Is sweat rate during steady state exercise related to maximum oxygen uptake? Journal of Thermal Biology, London, v.31, n.6, p.521-5, 2006.

BORG, G. Psychophysical bases of perceived exertion. Medicine and Science in Sports and Exercise, Hagerstown, v.14, p.377-81, 1982.

BUONO, M.J.; SJOHOLM, N.T. Effect of physical training on peripheral sweat production. Journal of Applied Physiology, Bethesda, v.65, p.811-4, 1988.

COTTER, J.D.; PATTERSON, M.J.; TAYLOR, N.A.S. The topography of eccrine sweating in humans during exercise. European Journal Applied Physiology, Berlin, v.71, p.549-54, 1995.

Sweat distribution before and after heat exposure. European Journal of Applied Physiology, Berlin, v.76, p.181-6, 1997. 
DUBOIS, D.; DUBOIS, E.F. A formula to estimate the approximate surface area if height and weight be known. Archives of Internal Medicine, Chicago, v.17, p.831-6, 1916.

EIKEN, O.; MEKJAVIC, I.B. Ischemia in working muscles potentiates the exercise-induced sweating responses in man. Acta Physiologica Scandinavica, Stockholm, v.181, p.305-11, 2004.

FALK, D. Brain evolution in Homo: the "radiator" theory. Behavioral Brain Science, Cambridge, v.13, p.333-81, 1990. FONSECA, I.A.T. Medida do conforto térmico durante as aulas de educaçáo física em climas tropicais. $2007.90 \mathrm{f}$. Dissertação (Mestrado em Educação Física) - Universidade Federal de Minas Gerais: Escola de Educação Física, Fisioterapia e Terapia Ocupacional, Belo Horizonte, 2007.

FORTNEY, S.M.; NADEL, E.R.; WENGER, C.B.; BOVE, J.R.. Effect of blood volume on sweating rate and body fluids in exercising humans. Journal of Applied Physiology: Respiratory Environmental Exercise Physiology, Bethesda, v.51, p.1594-1600, 1981.

HERTZMAN, A.B. Individual differences in regional sweating. Journal of Applied Physiology, Bethesda, v.10, p.242-8, 1957. HERTZMAN, A.B.; RANDALL, W.C.; PEISS, C.N.; SECKENDORF, R. Regional rates of evaporation from skin at various environmental temperatures. Journal of Applied Physiology, Bethesda, v.5, p.153-61, 1952.

INOUE, Y.; HAVENITH, G.; KENNEY, W.L.; LOOMIS, J.L.; BUSKIRK, E.R. Exercise- and methylcholine-induced sweating responses in older and younger men: effect or heat acclimation and aerobic fitness. International Journal Biometeorology, New York, v.42, p.210-6, 1999.

INOUE, Y.; NAKAO, M.; ARAKI, T.; MURAKAMI, H. Regional differences in the sweating responses of older and younger men. Journal of Applied Physiology, Bethesda, v.71, p.2453-9, 1991.

INOUE, Y.; NAKAO, M.; OKUDAIRA, S.; UEDA, H.; ARAKI, T. Seasonal variation in sweating responses of older and younger men. European Journal Applied Physiology, Berlin, v.70, p.6-12, 1995.

KONDO, N.; TAKANO, S.; AOKI, K.; SHIBASAKI, M.; TOMINAGA, H.; INOUE, Y. Regional differences in the effect of exercise intensity on thermoregulatory sweating and cutaneous vasodilation. Acta Physiologica Scandinavica, Stockholm, v.164, p.71-8, 1998.

KUNO, Y. Human perspiration. Springfield: Charles C. Thomas, 1956.

MACHADO-MOREIRA, C.A.; MAGALHĀES, F.C.; VIMIEIRO-GOMES, A.C.; LIMA, N.R.V.; RODRIGUES, L.O.

C. Effects of heat acclimation on sweating during graded exercise until exhaustion. Journal Thermal Biology, London, v.30, p.437-42, 2005.

MADEIRA, L.G. Efeitos das diferenças sexuais e da capacidade aeróbica sobre a sensibilidade e capacidade sudoríparas. 2007. 90 f. Dissertação (Mestrado em Educação Física) - Escola de Educação Física, Fisioterapia e Terapia Ocupacional, Universidade Federal de Minas Gerais, Belo Horizonte, 2007.

MORIMOTO, T.; SLABOCHOVA, Z.; NAMAN, R.K.; SARGENT II, F. Sex differences in physiological reactions to thermal stress. Journal of Applied Physiology, Bethesda, v.22, p.526-32, 1967.

NADEL, E.R.; BULLARD, R.W.; STOLWIJK J.A.J. Importance of skin temperature in the regulation of sweating. Journal of Applied Physiology, Bethesda, v.31, p.80-7, 1971.

NADEL, E.R.; MITCHELL, J.W.; SALTIN, B.; STOLWIJK, J.A.J. Peripheral modifications to the central drive for sweating. Journal of Applied Physiology, Bethesda, v.31, p.828-33, 1971.

NADEL, E.R.; PANDOLF, K.B.; ROBERTS, M.F.; STOLWIJK, J.A.J. Mechanisms of thermal acclimation to exercise and heat. Journal of Applied Physiology, Bethesda, v.37, p.515-20, 1974.

PATTERSON, M.J.; STOCKS, J.M.; TAYLOR, N.A.S. Humid heat acclimation does not elicit a preferential sweat redistribution towards the limbs. American Journal of Physiology, Bethesda, v.286, p.512-8, 2004.

PORTER, A.M.W. Sweat and thermoregulation in hominids: comments prompted by publications of P. E. Wheeler 1984-1993. Journal of Human Evolution, London, v.25, p.417-23, 1993.

RAICHLE, M.E. The brain's dark energy. Science, New York, v.314, p.1249-50, 2006.

ROSA, M.S. Comparaçáo das taxas de sudorese medidas em testes de campo realizados em ambiente tropical com a taxa de sudorese em cicloergômetro em ambiente laboratorial termoneutro. 2005. 110 f. Dissertação (Mestrado em Educação Física) - Escola de Educação Física, Fisioterapia e Terapia Ocupacional, Universidade Federal de Minas Gerais, Belo Horizonte, 2005. SÁ, K.R. Comparação entre a funçáo sudorípara de jovens masculinos e femininos durante exercício progressivo até exaustão. 2004. 77f. Dissertação (Mestrado em Educação Física) - Escola de Educação Física, Fisioterapia e Terapia Ocupacional, Universidade Federal de Minas Gerais, Belo Horizonte, 2005.

SATO, K.; DOBSON, R.L. Regional and individual variations in the function or the human eccrine sweat gland. The Journal of Investigative Dermatology, New York, v.54, p.443-9, 1970. 
SAWKA, M.N.; GONZALEZ, R.R.; YOUNG, A.J.; DENNIS, R.C.; VALERI, C.R.; PANDOLF, K.B. Control of thermoregulatory sweating during exercise in the heat. American Journal of Physiology, Bethesda, v.26, p.311-6, 1989. SHIBASAKI, M.; KONDO, N.; CRANDALL, C.G. Non-thermoregulatory modulation of sweating in humans. Exercise and Sport Sciences Reviews, Hagerstown, v.31, p.34-9, 2003.

SHIBASAKI, M.; WILSON, T.E.; CRANDALL, C.G. Neural control and mechanisms of eccrine sweating during heat stress and exercise. Journal of Applied Physiology, Bethesda, v.100, p.1692-1701, 2006.

TANKERSLEY, C.G.; SMOLANDER, J.; KENNEY, W.L.; FORTNEY, S.M. Sweating and skin blood flow during exercise: effects of age and maximal oxygen uptake. Journal of Applied Physiology, Bethesda, v.71, p.236-42, 1991. VIMIEIRO-GOMES, A.C.; MAGALHĀES, F.C.; AMORIM, F.T.; MACHADO-MOREIRA, C.A.; ROSA, M.S.; LIMA, N.R.; RODRIGUES, L.O.C. Comparison of sweat rate during graded exercise and the local rate induced by pilocarpine. Brazilian Journal Medicine and Biology Research, São Paulo, v.38 n.7, p.1133-9, 2005.

WEINER, J.S. The regional distribution of sweating. Journal of Physiology, London, v.104, p.32-40, 1945.

WEINMAN, K.P.; SLABOCHOVA, Z.; BERNAUER, E.M.; MORIMOTO, T.; SARGENT II, F. Reactions of men and women to repeated exposure to humid heat. Journal of Applied Physiology, Bethesda, v.22, p.533-8, 1967.

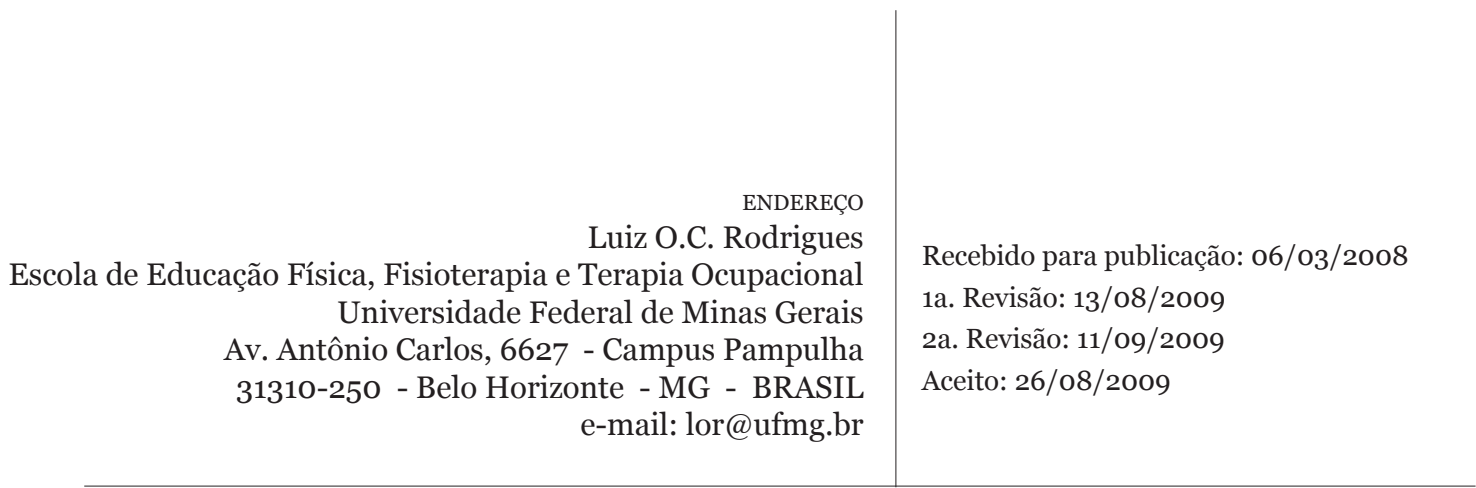

